\begin{tabular}{|c|l|}
\hline Title & ON THE ADSORPTION OF CARBON MONOX IDE ON PLA TINUM CA TALYST. \\
\hline Author(s) & KWAN, Takao \\
\hline Citation & JOURNAL OF THE RESEARCH INSTITUTE FOR CA TALY SIS HOKKA IDO UNIVERSITY, 1(2), 110-116 \\
\hline Issue Date & 1949-11 \\
\hline Doc URL & http://hdl.handle.net/2115/24605 \\
\hline Type & bulletin (article) \\
\hline File Information & 1(2)_P110-116.pdf \\
\hline
\end{tabular}

Instructions for use 


\section{ON THE ADSORPTION OF CARBON MONOXIDE ON PLATINUM CATALYST.}

By Takao Kwan.

For elucidating the mechanism of contact catalysis it is necessary to have information on the adsorbed state of the reactant. As regard to that of hydrogen on the metallic catalysts investigated hitherto by many workers the conclusive evidence has been obtained for the existance of hydrogen atoms on the catalyst's surface. As to the adsorbed state of the other reactants only few data are available even with such simple molecule as carbon monoxide or carbon dioxide.

Among these carbon monoxide might be one of the most interesting reactant both from the fundamental and industrial point of view, inasmuch as it yields by hydrogenation, notwithstanding so simple a structure, such variety of product at varying condition of the reaction, as those of Fischer-Tropsch synthesis for hydrogenation, methanol synthesis etc.

With regard to the former reaction KonAus and co-workers" proposed from the kinetical investigation that carbide was formed by the dissociation of carbon monoxide into carbon and oxygen atom in contradiction to Crax rort".

According to BEE $\mathrm{k}$ and co-workers" who observed the relative amount of adsorption of various gases to that of carbon monoxide on nickel film found that the latter is twice as large as that of hydrogen suggesting that carbon monoxide molecule as well as the dissociated hydrogen atom occupies one adsorption site on the surface or that carbon monoxide is adsorbed without dissociation.

The present author has previously investigated the adsorption of hydrogen on reduced nickel, cobalt and platinum and concluded having recourse to the statistical mechanical method that each of metal atoms on the catalyst's surface are equally capable of adsorbing a dissociated hydrogen atom ${ }^{1)}$. Extending the latter conclusion it may be assumed

1) Macsumura, Komsus and Taraya; J. Ind. Chem. Soc. Japan, 43 (1940) 420.

2) Crixmori); Trans. Far. Soc., 35 (1939) 946.

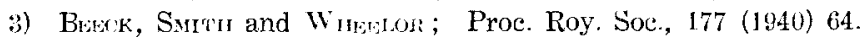

4) Kw.. and Ixu; "Catalyst" 4 (1943) w: 44 written in Japanese. 
On the Adsorption of Ca bon Monoxide on Plntinum Catalyst.

that every metal atom at the cataly st surface behaves physically identical even in the case of carbon monoxide adsorption.

On the basis of the assumption the adsorbed state of carbon monoxide on platinum catalyst is investigated in the present work by the similar method by determining the adsorption isotherm with special precaution for the thermodynamical equilibrium.

\section{Materials and Experimental Procedure}

Carbon monoxide; Carbon monoxide was prepared by dropping formic acid from the dropping funnel into an evacuated flask containing concentrated sulphuric acid at $90^{\circ}-100^{\circ} \mathrm{C}$ and passed throu a trap immersed in liquid air and then stored in a reservoir which was beforehand highly evacuated.

Platinum black; $0,93 \mathrm{gr}$ platinum black once used for the hydrogen adsorption was used here again.

The apparatus and the procedure for measurement of the adsorption was quite the same as those described in the foregoing paper.

\section{Experimental Results}

Measured quantity of carbon monoxide was admitted into the reaction vessel containing the platinum catalyst and maintained at a desired temperature. Although the adsorption rate was fairly rapid as in the case of hydrogen, the equilibrium pressure was determined about 20 hours after admission by approaching both from desorption and adsorption side similarly as in the previous case. Typical result is shown in Fig. 1 by the plot of the observed pressure against time elapsed along with occasionally varied temperature shown by annexed figures.

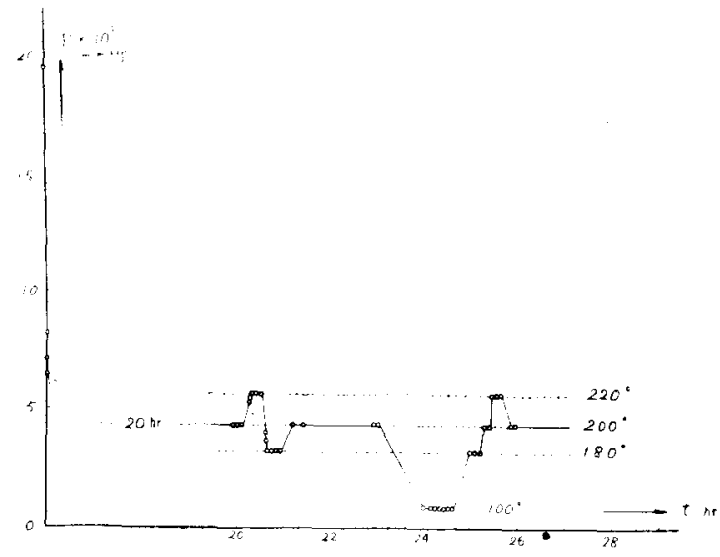

Fig. 1 Reversibility of carbon monoxide adsorption on platinum catalyst.

After the equilibrium was thus attained and appropriate pressure 
was read off additional portions of carbon monoxide are admitted successively into the reaction vessel and the corresponding equilibrium pressure were similarly determined in each case. The reversibility of the adsorption was assured over the whole range of adsorbed quantity although this was not the case with nickel or cobalt or iron at a lower adsorbed quantity..$^{\text {s) }}$

Calculating now the adsorbed quantity from the equilibrium pressure and the known quantity of admitted gas, adsorption isotherms were worked out at $180^{\circ}, 200^{\circ}, 220^{\circ}, 280^{\circ}$ and $300^{\circ} \mathrm{C}$ and at pressures lower than $1 \mathrm{~mm} \mathrm{Hg}$. The result is shown in Fig. 2.

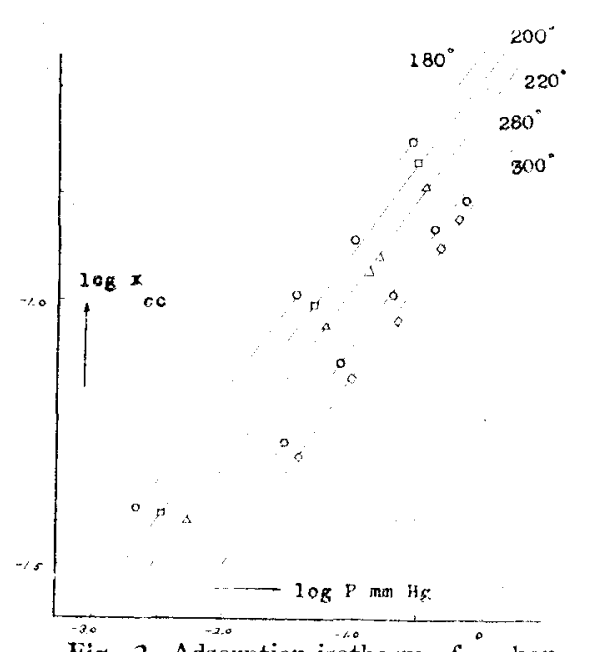

Fig. 2 Adsorption isotherm of carbon monoxide on platinum black.

The observed isotherms were found to be expressed by Freundlich's adsorption formulae as that,

$$
x=C P^{1 / n}, \quad n=2.7
$$

where $x$ is the adsorbed quantity and $P$ the equilibrium pressure.

The reported ready decomposition of carbon monoxide in the presence of metallic catalysts into carbon and carbon dioxide has not taken place as shown by the following control experiment.

The U-tube connected directly to the reaction vessel and immersed into liquid air during the adsorption measurement was now dipped after that into an alcohol bath cooled at -80 C. No pressure increases above that due to the relevant thermal expansion was observed showing that the evolution of carbon dioxide and hence the appropriate decomposition may be practically ignored in the present case.

Differential heat of adsorption $i \varepsilon$ was derived from the usual expression,

$$
\angle \varepsilon=R T^{2}\left(\frac{\partial \log P}{\partial T}\right)_{x}
$$

seperately from the isotherms at $280^{\circ} \mathrm{C}$ and $300^{\circ} \mathrm{C}$ on one hand and from those at $180^{\circ}, 200^{\circ}$ and $220^{\circ} \mathrm{C}$ on the other hand inasmuch as the catalyst

5) KWN and IWv ; "Catalyst" 5 (1949) 43. 
was treated with hydrogen at $350^{\circ} \mathrm{C}$ between the observation of the former group of isotherms and that of the latter one and such procedure is known by experience liable to disturb the reproducibility of the cataiyst's activity. The results are shown in the Table below.

As shown by the Table $\Delta s$ is affected neither by the temperature range at which it is determined nor

Trume 1 Heat of adsorption of carbon monoxide on platinum catalyst (Kcalimole)

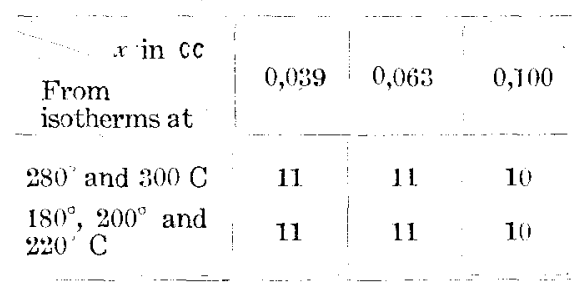

\section{Statistical Mechanical Treatment}

Adsorption isotherm of diatomic molecules $\hat{\partial}$ formed by atoms $\hat{\partial}_{1}$ and $\partial_{2}$. is statistical mechanically derived introducing a set of function $p, q$ and $\theta$ due to Horict ${ }^{\text {s) }}$ as follows ;

$$
\begin{aligned}
& \frac{\theta}{1-\theta}=\frac{q^{\bar{j} a}}{p^{\bar{j}(a)}} \\
& \begin{array}{c}
\theta \\
1-2 \theta
\end{array}=\frac{q^{\delta_{1}(a)}}{p^{\left.\delta_{1} a\right)}}=\begin{array}{l}
q^{\delta_{2}(a) *} \\
p^{\delta_{2}(a)}
\end{array}
\end{aligned}
$$

where $\delta(\alpha)$ denotes the molecule $\delta, \partial_{i}(\alpha)$ and $\partial_{(}(\alpha)$ dissociated atoms respectively at the absorbed state.

At adsorption equilibrium we have that,

$$
\begin{aligned}
& p^{\bar{\delta}}=p^{\grave{j}(a)} \\
\text { or } \quad p^{\bar{\delta}} & =p^{\delta_{i}^{\prime}(\boldsymbol{a})} p^{\delta_{4}(\boldsymbol{a})}
\end{aligned}
$$

respectively for the case when $\partial$ is undissociated or dissociated at the adsorbed state. In the former case we have from Eq. (1.1) and (2.1) as that,

$$
\frac{\theta}{1-\theta}=\frac{q^{\check{\delta}(\boldsymbol{a})}}{p^{\Sigma}}
$$

and in the latter as that,

$$
\left(\begin{array}{c}
\theta \\
1-2 \theta
\end{array}\right)^{2}=\begin{gathered}
q^{\delta_{1}(a)} q^{\delta_{2}^{(a)}} \\
p^{\delta}
\end{gathered}
$$

*) The $\theta$ must be equal because of the stoichiometrical relation for $\delta_{1}$ and $\delta_{\text {. }}$.

5) Hounth; J. Res. Inst. Catalysis, This Volume, (1948) 8. 


\section{- Journal of the Research Institute for Catalysis}

Since $p^{\delta}$ is however denoted from it's definition as that,

$$
p^{\delta}=\frac{Q^{\delta}}{N^{\delta}}=\frac{k T Q^{\delta}}{P}
$$

where $P$ is the pressure and $Q$ the partition function, Eq. (3.1) and (3.2) are now respectively expressed as that,

$$
\begin{gathered}
\frac{\theta}{1-\theta}=\frac{q^{\delta(a)} P}{l i T Q^{\delta}} \\
\left(\frac{\theta}{1-2 \theta}\right)^{=}=\frac{q^{\delta_{1}^{(a)}} q^{\delta^{j}(a)} P}{k T Q^{j}}
\end{gathered}
$$

Eqs. (5.1) and (5.2) are further transformed in a suitable form for calculation respectively as that,*)

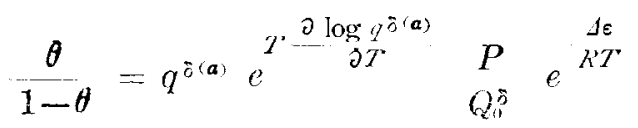

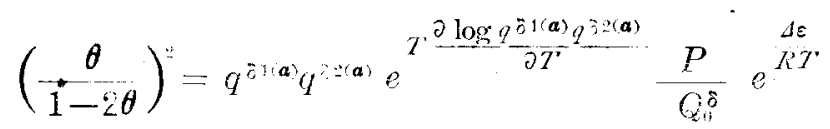

where

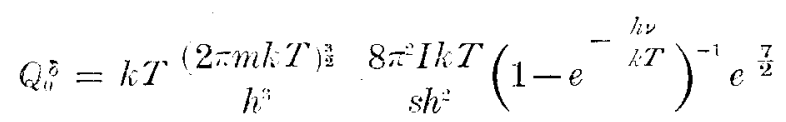

$$
\begin{aligned}
& h \text {; Planck constant } \\
& \text { k; Boltzmann constant } \\
& s \text {; Symmetry number }
\end{aligned}
$$

$m, I$ and $\nu$; Mass, moment of inertia and fundamental frequency of gaseous molecule $i$.

$q^{\delta(a)}, q^{\delta_{1}^{\prime(a)}}$ and $q^{\delta_{1}(a)}$; Function particular to the adsorbed state of $\delta(\alpha)$,

$$
\grave{o}_{1}(a) \text { and } \grave{o}_{i}(a) \text { denoted as } q^{\hat{o}_{1}(a)}=i\left(I-e^{-\frac{h \Sigma \nu_{i}}{k T}}\right)^{-1} e^{-\frac{\varepsilon_{0}+\frac{1}{2} h \Sigma_{\nu_{i}}}{k_{i}}} \text { etc. }
$$

where $\nu_{i}^{\prime}$ 's are frequency of normal vibration at the adsorbed state.

Taking now $\hat{o} \equiv C O$ and assuming $h \nu_{\varepsilon} \geqslant k T$ in $q$ 's and hence

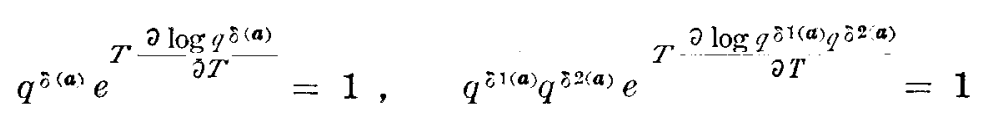

*Cf. the foot note on P. 93. 
On the Adsorption of Carbon Monoxide on Platinum Catalyst.

the calculation of the covered fraction of the surface was carried out at temperature $300^{\circ} \mathrm{C}$ and at pressures 0,01 and $0,1 \mathrm{~mm} \mathrm{Hg}$ using the observed heat of adsorption $11 \mathrm{Kcal}$ mole and the following molecular constants of carbon monoxide molecule respectively for Eq. (6.1) and (6.2).

$$
I=14,37 \times 10^{-+11} \quad \text { g. } \mathrm{cm}^{*} * \quad \nu=2168,80 \mathrm{~cm}^{-1} *
$$

On the other hand the covered fraction of the surface $\theta_{\text {ohs }}$ was determined from the adsorbed quantity and the surface area of the catalyst assuming every platinum atom on the surface equally capable of adsorbing carbon or oxygen atom.

The particle size of platinum black estimated by the diffuseness observation of the Debye-Scherrer pattern of the electron diffraction was found, as mentioned in the previous paper', appreciably smaller than that given by B.E.T. method although in the case of nickel the particle size estimated by either of the methods was found satisfactorily coincident with each other. Without inquiring into the above discrepancy $\theta_{\text {,in }}$ was calculated on the basis of $280 \times 10^{4} \mathrm{~cm}^{\prime \prime} / \mathrm{gr}$ or the particie size $10 \AA$ due to the electron diffraction method as well as of $14 \times 10^{\prime} \mathrm{cm}^{\prime \prime} / \mathrm{gr}$ due to B.E.T. method assuming that $10^{\prime} \mathrm{Pt}$ atoms per square centimeter are capable of adsorbing each one carbon or oxygen atom. The results are shown together with those given by statistical mechanical calculation in the following Table.

Tamis 2 The relation between the equilibrium pressure $P$ and the covered fraction $T=300^{\circ} \mathrm{C} \quad<\varepsilon=11 \mathrm{Kcal} / \mathrm{mole}$

\begin{tabular}{|c|c|c|c|}
\hline$\theta$ & $\begin{array}{l}\text { quil. Pressure } \mathrm{mm} \mathrm{Hg} \\
\text { mark }\end{array}$ & 0,01 & 0,1 \\
\hline$\theta_{\text {calc }}$ & $\begin{array}{l}C O \rightleftarrows C O(a) \\
C O \rightleftarrows C(a)+O(a)\end{array}$ & $\begin{array}{l}10^{-12} \\
10^{-6}\end{array}$ & $\begin{array}{r}10^{-13} \\
3 \times 10^{-6}\end{array}$ \\
\hline$\theta_{\mathrm{obs}}$ & $\begin{array}{l}\text { Electron diffraction } \\
\text { B. E. T. }\end{array}$ & $\begin{array}{l}3 \times 10^{-4} \\
6 \times 10^{-3}\end{array}$ & $\begin{array}{r}7 \times 10^{-4} \\
10^{-2}\end{array}$ \\
\hline
\end{tabular}

As seen from the Table assumed adsorbed state of $C O(\alpha)$ fails exclusively to account for the experiment whereas $C(\alpha)+O(a)$ or the

*) Sroner; Molekulspektren, Berlin 1936.

6) KWAN; This Journal p. 89 
dissociated state fits in with regard to the accuracy of the basic data; in the case of undissociated adsorption $\theta_{0 b}$ shuld be different according as earbon monoxide occupies one or two $\mathrm{Pt}$ atoms but this makes no difference with the above conclusion.

Conclusion is that carbon monoxide is adsorbed on platinum being dissociated into carbon and oxygen atom.

In conclusion, the present author wish to express my sincere thanks to the Director of the Institute J. Honirrr for his kind interest and valuable discussion on this work.

The Research Institute for Catalysio Hokkaido University

Sapporo, Japan 